\title{
Retrospective Analysis of Pediatric Alopecia Areata Treated with Methotrexate
}

\author{
Emily Correia, $B S^{1}$, Michael Jones ${ }^{2}$ and Leslie Castelo-Soccio, MD, PhD ${ }^{3 *}$ \\ ${ }^{1}$ Sidney Kimmel Medical College, Thomas Jefferson University, USA \\ ${ }^{2}$ Department of Biostatistics and Management Core, The Children's Hospital of Philadelphia, USA \\ ${ }^{3}$ Division of Pediatrics, Section of Dermatology, The Children's Hospital of Philadelphia, USA
}

\begin{abstract}
Background: Therapy for alopecia areata (AA) in pediatric patients is challenging and unstandardized. Common treatments for alopecia include topical agents, triamcinolone injections, and systemic agents. Treatment using systemic agents in children is controversial due to possible negative side effects and the need for long term therapy because of potential relapse after cessation. The purpose of this study was to evaluate the efficacy and safety of methotrexate therapy for the treatment of AA in pediatric patients. This medication is affordable and routinely used in children for other chronic conditions.
\end{abstract}

Methods: This retrospective chart review included patients treated with methotrexate for AA who were managed by the Dermatology Section at the Children's Hospital of Philadelphia between 2015 and 2018. The following data was extracted from patients' charts: severity of disease measured by Severity of Alopecia Tool (SALT) scores, duration of treatment course, side effects, age, race, and sex.

Results: 27 pediatric patients treated with methotrexate were included in the study. There was a statistically significant decrease in SALT score between initial SALT score and score at 12-15 months, but not between initial score and SALT at 6-9 months. Mood changes and gastrointestinal discomfort were the most commonly reported adverse effects.

Conclusion: Our study demonstrates that patients treated with methotrexate had a statistically significant improvement in SALT scores at 12-15 months, despite the SALT scores showing no improvement at 6-9 months. This suggests the results of methotrexate may be delayed, but that the mediation is still effective and safe.

\section{Introduction}

Alopecia areata (AA) is a chronic disease in which T-cell mediated autoimmune processes cause non-scarring patchy hair loss [1]. It has been previously reported that AA's lifetime incidence is approximately $1.7 \%$ worldwide, but more recent studies estimate it to be approximately $2.1 \%$ [2-4]. In the United States between the years 1990 and 2000, this amounted to 2.4 million office visits related to AA [5]. Alopecia areata has deep impacts on quality of life and individuals with alopecia are at increased risk of depression and anxiety [2]. There are no FDA approved therapies for Alopecia areata and current treatments work for some, but not all, patients.

The severity of alopecia can vary between individuals in number and size of patches of hair loss. In addition to patchy hair loss, alopecia can involve total loss of scalp hair, known as alopecia totalis (AT), or involve loss of all body hair, known as alopecia universalis (AU) [6]. Common treatments for alopecia include topical agents such as corticosteroids, minoxidil, irritants, and allergens, as well as ultraviolet lightbased therapies and localized injections with triamcinolone.
Additionally, systemic therapies include oral corticosteroids, methotrexate, biologics, and off label JAK kinase inhibitors such as tofacitinib or biologics like dupilumab, currently in trial as a treatment for AA $[7,8]$.

An Australian consensus created guidelines to direct the management of $\mathrm{AA}$. This consensus determined that the mainstay of therapy for most presentations of alopecia are corticosteroid injections for both adults and topical corticosteroids or immunotherapies for children. However,

*Corresponding author: Leslie Castelo-Soccio, MD, PhD, Division of Pediatrics, Section of Dermatology, Children's Hospital of Philadelphia; 3401 Civic Center Blvd, 3rd Floor Dermatology, Philadelphia, PA 19104, USA

Accepted: July 21, 2021

Published online: July 23, 2021

Citation: Correia E, Jones M, Castelo-Soccio L, et al. (2021) Retrospective Analysis of Pediatric Alopecia Areata Treated with Methotrexate. Dermatol Arch 5(1):127-130 
with rapidly progressing or extensive disease, systemic therapies, such as oral methotrexate, are encouraged [9].

Small studies have been published on the use of methotrexate for AA with promising results. A systematic review and meta-analysis revealed that methotrexate is reasonably effective in patients with severe $A A$, but that adults are more responsive to treatment than pediatric patients [10-14]. Furthermore, an updated meta-analysis for pediatric patients with AA on methotrexate showed success rates of $49.7 \%$, recurrence rates of $30 \%$ and side effects in only $15.7 \%$ of cases. However the largest sample size encountered included 14 children [15]. Therefore, more information about this therapy should be investigated in order to make reasonable conclusions about using methotrexate in pediatric patients.

$A$ recent case series on pediatric patients demonstrated a $70 \%$ success rate of methotrexate for $A A$ with no serious side effects, other than nausea and vomiting [15]. Another study conducted on children with AA reported regrowth with methotrexate in over half of the patients studied, demonstrating that methotrexate is an effective medication in pediatric AA. The study also suggests that it is generally safe, as no cases discontinued the medication due to side effects [16]. However, it is difficult to make clear conclusions about methotrexate therapies since sample sizes have been limited. Therefore, more studies must be conducted.

Methotrexate use in pediatric patients is commonly disputed due to concern for side effects. Not only is methotrexate immunosuppressive, but it can also cause gastrointestinal upset, raise liver enzymes, and produce hematological abnormalities [17]. These effects precipitate controversy around the use of methotrexate, so its safety must be evaluated further.

Here we report a retrospective chart review conducted at Children's Hospital of Philadelphia of 27 pediatric patients with AA treated with methotrexate. The Severity of Alopecia Tool (SALT) Score, time to relapse, and secondary side effects were assessed in these subjects.

\section{Methods}

With institutional review board approval, we conducted a retrospective chart review of pediatric and adolescent patients ages 1-18 years at the Children's Hospital of Philadelphia with a clinical diagnosis of Alopecia Areata, Alopecia Totalis, or Alopecia Universalis who were treated with oral methotrexate therapy for AA from 2015 to 2018. Demographic data for each patient including sex, race/ethnicity, and age at treatment commencement were recorded when available (Table 1).

Dosage of oral methotrexate and duration of treatment were documented for enrolled subjects. Systemic treatment was often combined with topical therapies and/or intralesional triamcinolone acetonide injections. Additionally, folate supplementation was prescribed for the duration of treatment.

The following were recorded: Severity of Alopecia Tool (SALT) scores at the start of treatment as well as at intervals
Table 1: Study subject demographics.

\begin{tabular}{|l|c|c|c|}
\hline Demographic & & & \\
\hline Sex & $\mathbf{n}$ & $\%$ & \\
\hline Male & 12 & 44.4 & \\
\hline Female & 15 & 55.6 & \\
\hline Race & & & \\
\hline White & 14 & 51.9 & \\
\hline Other & 13 & 48.1 & \\
\hline Black & 1 & 3.7 & \\
\hline Asian & 2 & 7.4 & \\
\hline Indian & 2 & 7.4 & \\
\hline Other & 6 & 22.2 & \\
\hline Refused & 1 & 3.7 & \\
\hline American Indian or Alaska native & 1 & 3.7 & \\
\hline Age in years & min & Max & mean \\
\hline & 5 & 17 & 12.2 \\
\hline
\end{tabular}

during the course of treatment. Improvement was measured by changing SALT scores where $0 \%$ means no hair loss and $100 \%$ means no hair on the scalp. The difference between SALT scores at initiation of treatment and final visit was calculated in order to note the overall effect of the therapeutic agent. Patients were analyzed at start of treatment (Time 1), 6-9 months (Time 2), and 12-15 months (Time 3). It was documented whether patients were continued on methotrexate at the time of the review, and the therapy chosen if methotrexate therapy was discontinued.

Statistical analysis was performed by the Children's Hospital of Philadelphia Biostatistics and Management Core. In analyzing treatment efficacy, SALT scores were the primary metric. A Generalized Estimating Equations (GEE) model using a Gamma link function was used to observe the association between SALT and Time. Wilcoxon Signed-Rank tests were used to evaluate the difference in SALT scores from baseline versus each consecutive time point, using a Bonferroni $p$-value adjustment for multiple comparisons. Furthermore, a paired T-test was used to determine if the difference in average SALT scores between Time 1 and Time 3 differed from zero for the patients. After correction, significance was considered for $p$-value $<0.01$. Analysis of side effects for patients on methotrexate was conducted using the percent of patients that reported each side effect.

\section{Results}

27 patients were included in this study, however SALT scores were only consistently calculated for 21 of these patients due to loss to follow up or missed appointments. The average age of subjects on methotrexate was 12.2 years with a minimum of 5 years and a maximum of 17 years.

The effect of time on average SALT scores for patients on methotrexate using Time 1 as the reference point was analyzed by using GEEs. The average SALT score reduction between Time 1 and Time 2 failed to show statistical significance, however the difference between the averages at Time 1 and Time 3 reaches statistical significance ( $p$-value < 0.0001) (Table 2). The average difference in SALT scores from the start of the study to $12-15$ months was calculated to be 
Table 2: Summary statistics of SALT scores at baseline and each subsequent time point for methotrexate.

\begin{tabular}{|c|c|c|c|c|c|}
\hline \multicolumn{1}{|c|}{ Time } & Estimate & $\begin{array}{c}\text { Standard } \\
\text { Error }\end{array}$ & \multicolumn{2}{|c|}{ Confidence Limits } & p-value \\
\hline SALT at $\mathrm{T}_{1}$ & 74.2381 & 6.6041 & 61.2943 & 87.1819 & $<0.0001$ \\
\hline SALT at $\mathrm{T}_{2}$ & -0.4524 & 8.3525 & -16.8231 & 15.9183 & 0.9568 \\
\hline SALT at $\mathrm{T}_{3}$ & -44.3810 & 11.2850 & -66.4992 & -22.2627 & $<0.0001$ \\
\hline
\end{tabular}

The estimate signifies the average baseline SALT at T1 and the difference in SALT at the subsequent times. This table ignores possible confounders. The confidence limits estimates the range of possible values for the parameter and the standard error is the standard deviation of the distribution from the sample.

10.9, indicating that the final SALT scores were lower than at the start of treatment. This difference is statistically significant ( $p$-value $=<0.0001)$.

Additional covariates were tested in the presence of time for methotrexate that included age at onset, sex, weight and race. The significance of time does not change when adding any of the possible confounders. However, the average SALT score for males was statistically significantly higher than the SALT score for females ( $p$-value $=0.0063$ ). Additionally, the average SALT score for white patients was higher when compared to subjects of other races ( $p$-value $=0.0468$ ).

Adverse effects and lab abnormalities while on methotrexate were also observed and analyzed. Mood changes (63.6\%) were the most prominent side effect noted, with $\mathrm{Gl}$ discomfort being the second most common side effect (22.7\%). However, $22.2 \%$ of the subjects reported no side effects at all. Other side effects reported included fatigue, headache, blurry vision, and decreased appetite. 15 patients had a bloodwork abnormality while 12 patients had no abnormalities. The most common blood abnormalities included transient elevated transaminases, decreased alkaline phosphatase, decreased mean platelet volume, increased eosinophils, increased glucose, and decreased hemoglobin.

\section{Discussion}

While there are no approved FDA treatments approved for alopecia, an effective and safe medication for AA must be investigated due to the detrimental effects that the disease may have on a patient's quality of life. Methotrexate may be a successful treatment modality, but efficacy in children must be studied further. This report provides evidence for the safety of methotrexate in treating pediatric $A A$ and may give hope to patients struggling with the disease.

The data in this study demonstrates that SALT scores had a statistically significant difference between Time 1 and Time 3, but not between Time 1 and Time 2, suggesting that the efficacy of methotrexate may be delayed and response may depend on the length of treatment. Therefore, methotrexate may be a possible long-term treatment for AA considering the statistically significant improved SALT score that occurred with a longer duration of treatment. Ideally, a longer trial of methotrexate use with a larger sample size could enhance our understanding of these preliminary findings.
Mood change was the most commonly reported adverse effect during methotrexate therapy, with one subject discontinuing therapy. This outcome is different from previous results on methotrexate use in adults with alopecia areata and pediatric patients; these studies declare that gastrointestinal adverse events were most common [10,15]. However, it cannot be concluded whether mood changes are due to the medication. The psychological impact of the disease, along with natural emotional changes that occur during maturation, may have also played a part in reporting this side effect. Additionally, gastrointestinal symptoms were also commonly noted in our patients. Of note, many subjects on methotrexate also reported no adverse events or very minimal side effects, therefore the use of methotrexate may still be considered safe in pediatric patients.

However, some limitations to this study exist. Because of the lack of control group and use of concomitant topical medication or steroid injections, the decrease in SALT scores cannot be confidently attributed to methotrexate treatment. This change may be due to the natural course of the disease that consists of resolution and relapse of alopecia flares, or the concomitant treatments. Additional limitations of this study include small sample size, left skewed SALT scores, and non-normal distribution of baseline SALT scores. Despite this being largest study in the current literature, an even larger sample size would be necessary to potentially observe subtle benefits methotrexate as a treatment in pediatric patients.

Although long-term treatment of AA is often unsuccessful, this study demonstrates that systemic therapies such as methotrexate may play an important role in the treatment regimen for pediatric patients with more severe $A A$, as evidenced by SALT score changes at certain time points. Furthermore, despite the fact that there are many studies conducted on adults, those results cannot be applied to children and more pediatric studies are important to determine safe and effective therapies for children. To our knowledge, this is the largest cohort of pediatric patients on methotrexate for AA reported in the literature. Additional studies investigating dose, length of treatment, treatment combinations, and patient selection will be essential in optimizing therapeutic regimens for future patients.

\section{Acknowledgments}

None

\section{Declarations}

\section{Funding}

None

\section{Conflicts of interest}

The authors have no conflicts of interest to disclose

\section{Availability of data and material}

All data in this study is original and will be freely available on request from the authors to any researcher wishing to use for non-commercial purposes 


\section{Author's Contributions}

Emily Correia, BS: Conceptualization, acquisition and analysis of data, writing, review and editing, final approval for publication.

Michael Jones: Analysis of data, final approval for publication.

Leslie Castelo-Soccio, MD, PhD: Conceptualization, review and editing, final approval for publication.

\section{References}

1. Spano F, Donovan JC (2015) Alopecia areata: Part 1: Pathogenesis, diagnosis, and prognosis. Can Fam Physician 61: 751-755.

2. Villasante Fricke AC, Miteva M (2015) Epidemiology and burden of alopecia areata: A systematic review. Clin Cosmet Investig Dermatol 8: 397-403.

3. Safavi KH, Muller SA, Suman VJ, (1995) Incidence of alopecia areata in Olmsted County, Minnesota, 1975 through 1989. Mayo Clin Proc 70: 628-633.

4. Mirzoyev SA, Schrum AG, Davis MDP, et al. (2014) Lifetime incidence risk of alopecia areata estimated at $2.1 \%$ by Rochester epidemiology project, 1990-2009. J Invest Dermatol 134: 11411142.

5. McMichael AJ, Pearce DJ, Wasserman D, et al. (2007) Alopecia in the United States: Outpatient utilization and common prescribing patterns. J Am Acad Dermatol 57: S49-S51.

6. Pratt $\mathrm{CH}$, King LE, Messenger AG, et al. (2017) Alopecia areata. Nature Reviews Disease Primers 3: 17011.

7. Renert-Yuval Y, Guttman-Yassky E (2017) The changing landscape of alopecia areata: The therapeutic paradigm. Adv Ther 34: 1594-1609.
8. Ramot Y, Zlotogorski A (2020) JAK Inhibitors for the Treatment of Alopecia Areata. Harefuah 159: 38-42.

9. Cranwell WC, Lai VW, Photiou L, et al. Treatment of alopecia areata: An Australian expert consensus statement. Australas J Dermatol 60: 163-170.

10. Van ATT, Lan AT, Anh MH, et al. (2019) Efficacy and safety of methotrexate in combination with mini pulse doses of methylprednisolone in severe alopecia areata. The vietnamese experience. Open Access Maced J Med Sci 7: 200-203.

11. Luggen $P$, Hunziker $T$ (2008) High-dose intravenous corticosteroid pulse therapy in alopecia areata: Own experience compared with the literature. J Dtsch Dermatol Ges 6: 375-378.

12. Friedland R, Tal R, Lapidoth M, et al. (2013) Pulse corticosteroid therapy for alopecia areata in children: A retrospective study. Dermatology 227: 37-44.

13. Lalosevic J, Gajic-Veljic M, Bonaci-Nikolic B, et al. (2015) Combined oral pulse and topical corticosteroid therapy for severe alopecia areata in children: A long-term follow-up study. Dermatol Ther 28: 309-317.

14. Phan K, Ramachandran V, Sebaratnam DF (2019) Methotrexate for alopecia areata: A systematic review and meta-analysis. J Am Acad Dermatol 80: 120-127.

15. Phan K, Lee G, Fischer G (2020) Methotrexate in the treatment of paediatric alopecia areata: Retrospective case series and updated meta-analysis. Australas J Dermatol 61: 119-124.

16. Landis ET, Pichardo-Geisinger RO (2018) Methotrexate for the treatment of pediatric alopecia areata. J Dermatolog Treat 29: 145-148.

17. Desai C (2016) Meyler's side effects of drugs: The international encyclopedia of adverse drug reactions and interactions. Indian J Pharmacol 48: 224-225.

DOI: $10.36959 / 661 / 318$

Copyright: (c) 2021 Correia E, et al. This is an open-access article distributed under the terms of the Creative Commons Attribution License, which permits unrestricted use, distribution, and reproduction in any medium, provided the original author and source are credited. 